\title{
LA LIBERTAD DE CÁTEDRA EN ALEMANIA
}

\author{
HARTMUT KRÜGER \\ Profesor ordinario de Derecho Público \\ en la Universidad de Colonia
}




\section{SUMARIO}

I. Evolución histórica de la libertad de cátedra. II. La libertad de cátedra. 1. Concepto y fundamento de la libertad de cátedra. 2. Ámbito de la libertad de cátedra. 3. Planificación, coordinación y organización de la actividad docente. 4. La reserva de la cláusula de fidelidad. III. CuESTIONES JURÍDICAS SOBRE LA EVALUACIÓN DE LA ENSEÑANZA. 


\title{
LA LIBERTAD DE CÁTEDRA EN ALEMANIA ${ }^{1}$
}

\author{
POR \\ HARMUT KRÜGER \\ Profesor ordinario de Derecho Público \\ en la Universidad de Colonia
}

\section{NOTA INTRODUCTORIA}

El texto que se ofrece a continuación es la traducción al castellano del capítulo sobre libertad de cátedra escrito por el profesor Hartmut Krüger en el Manual de Derecho de las Ciencias publicado en $1996^{2}$. Me parece que el texto es una excelente exposición sobre el concepto, alcance y regulación de la libertad de cátedra en Alemania. Por ello, creo de todo interés el que se conozca su contenido por parte de los estudiosos de este problema.

Quiero además ofrecer esta traducción como homenaje al prof. Krüger, con quien he trabajado durante 1998 , hasta su inesperado y repentino fallecimiento, el 8 de julio de dicho año. Los profesores Stern y Leuze han mostrado recientemente el pesar que entre los juristas alemanes ha producido esta prematura desaparición, $y$ han puesto de relieve en sendos artículos la valía científica y académica de Hartmut Krüger ${ }^{3}$.

1 Traducción y Nota introductoria de Carlos Vidal Prado, profesor Asociado del Departamento de Derecho Constitucional de la UNED.

2 KRÜGER, Hartmut, "Lehre", en Handbuch des Wissenschaftsrechts (Editores: Flämig, Kimminich, Krüger, Meusel, Rupp, Scheven, Schuster, Graf StenbockFermor), segunda edición, Springer, Berlin, 1996, pp. 309-322.

3 STERN, K., "Professor Dr. Hatmut Krüger verstorben", en Kölner Universitäts Journal, Ausgabe 3-1998, pp. 60-62; Leuze, D., "Hartmut Krüger (30.August 1943-8.Juli 1998)", en WissR, Bd. 31 (1998), pp. 211-213. 
Nacido en Greifswald en 1943, el prof. Krüger realizó sus estudios de Derecho en las Universidades de Friburgo y Kiel. Fue promovido al grado de Doctor en la Universidad de Kiel en febrero de 1972. En 1973 se incorporó a la Universidad de Regensburg, como Asistente del prof. Otto Kimminich, en la Cátedra de Derecho Público. Allí alcanzó su Habilitation para Derecho Político y Administrativo (Staats- und Verwaltungsrecht). Después de desempeñar actividades docentes en las Universidades de Colonia y Gotinga, fue llamado a ocupar una plaza de Profesor de Derecho Público en la Universidad de Colonia en octubre de 1986, donde a partir de 1994 dirigía el Instituto de Derecho de las Ciencias (Institut für Wissenschaftsrecht).

Quizá por influencia del prof. Kimminich, la producción científica de Hartmut Krüger se centra sobre todo en el ámbito del Derecho universitario o de las Escuelas Superiores (Hochschulrecht), y especialmente en el Derecho de las Ciencias (Wissenschaftsrecht). Numerosas publicaciones en estos ámbitos así lo acreditan, comenzando por su trabajo de Habilitation en Regensburg, y, en los últimos años, con su labor al frente de la revista de Derecho de las Ciencias (Wissenschaftsrecht, Wissenschaftsverwaltung, Wissenschaftsförderung), y la publicación del Manual citado al inicio de estas líneas, del cual fue también editor.

Como ha dicho el prof. Stern, estos intereses científicos «movieron al prof. Krüger a proponer con insistencia junto con otros colegas la creación de un Instituto de Derecho de las Ciencias alemán y europeo ${ }^{4}$ en la Universidad de Colonia, meta que alcanzó en 1994, siendo nombrado director de dicho Instituto. El Institut für Deutsches und Europäisches Wissenschaftsrecht -único en Alemania- ha alcanzado en estos años un notable prestigio por su labor de profundización y estudio de los problemas jurídicos que afectan al ámbito universitario y científico. El prof. Krüger tuvo ocasión además de llevar a la práctica sus investigaciones, al asesorar frecuentemente a políticos y gestores en el ámbito educativo y universitario. En concreto, como ha resaltado el prof. Leuze, durante años fue presidente del Claustro académico de la Universidad de Colonia, precisamente en el tiempo en que se reformó el Ordenamiento jurídico de dicha Universidad, labor en la cual participó activamente ${ }^{5}$.

Su preferencia por el Derecho de las Ciencias no fue óbice, sin embargo, para que el prof. Krüger profundizase en otros campos del

4 Stern, K., op. cit., p. 62.

5 LeUZE, D., op. cit., p. 212. 
Derecho Público, como el Derecho Constitucional y Administrativo (en especial el Derecho de los Funcionarios Públicos, Beamtenrecht), así como el Derecho Comunitario.

\section{ABREVIATURAS}

Abs.

Art.

BayVGH

Beiheft

Bd.

BT-Drucks

BVerfG

BVerfGE

BVerwGE

DÖV

DVBI

ESVGH

GG

HRG

HessVGH

JöR N.F.

$J Z$ Absatz (párrafo). Artículo.

Sammlung von Entscheidungen des bayerischen Verwaltungsgerichtshofes (Recopilación de la jurisprudencia del Tribunal Superior Administrativo de Baviera). Suplemento.

Band (Tomo).

Drucksachen des Deutschen Bundestages (Publicaciones del Parlamento alemán).

Bundesverfassungsgericht (Tribunal Constitucional Federal).

Amtliche Sammlung der Entscheidungen des Bundesverfassungsgerichts (Recopilación oficial de la Jurisprudencia del Tribunal Constitucional).

Entscheidungen des Bundesverwaltungsgerichts (Jurisprudencia del Tribunal Administrativo Federal).

Die öffentliche Verwaltung (La Administración Pública). Deutsches Verwaltungsblatt (Revista Alemana de la Administración).

Entscheidungssammlung. Hessischer Verwaltungsgerichtshof, Verwaltungsgerichtshof Baden-Württemberg (Recopilación de jurisprudencia. Tribunal Superior Administrativo del Estado de Hessen, Tribunal Superior Administrativo del Estado de Baden-Württemberg). Grundgesetz (Ley Fundamental de Bonn). Hochschulrahmengesetz (Ley Marco de Universidades). Verwaltungsgerichtshof (Tribunal Superior Administrativo) de Hesse.

KMK-HSchR Informationen zum Hochschulrecht. Veröffentlichungen der Kulturministerkonferenz (Información sobre Derecho de universidades. Publicaciones de la Conferencia de Ministros de Cultura). 
NJW

Neue Juristische Wochenschrift (Nueva Revista Jurídica semanal).

NVwZ Neue Zeitschrift für Verwaltungsrecht (Nueva Revista de Derecho Administrativo).

NWVBI Nordrhein-Westfälische Verwaltungsblätter (Boletín Administrativo de Renania del Norte-Westfalia).

OVG Berlin Oberverwaltungsgericht (Tribunal Superior Administrativo) de Berlín.

OVG Lüneburg Oberverwaltungsgericht (Tribunal Superior Administrativo) de Niedersachsen (Lüneburg).

OVG NW Oberverwaltungsgericht (Tribunal Superior Administrativo) de Renania del Norte Westfalia (Münster).

Rdnr. Randnummer (número marginal).

UG NW Universitätsgesetz Nordrhein-Westfalen (Ley Universitaria de Renania del Norte-Westfalia).

VerwRspr Verwaltungsrechtsprechung in Deutschland (Jurisprudencia administrativa en Alemania).

VG Verwaltungsgericht (Tribunal Administrativo).

VGH BW Verwaltungsgerichtshof Baden-Württemberg (Tribunal Superior Administrativo) de Baden-Württemberg.

WissR

Wissenschaftsrecht, Wissenschaftsverwaltung, Wissenschaftsförderung (Revista de Derecho de las Ciencias).

\section{EVOLUCIÓN HISTÓRICA DE LA LIBERTAD DE CÁTEDRA}

Antes de la reforma universitaria de Wilhelm von Humboldt $t^{6}$ encontramos ya en Alemania los orígenes históricos de la libertad de cátedra tal como hoy es concebida.

La «libertas philosophandi", por ejemplo, fue otorgada como privilegio a Thomasius -que había huído de Leipzig a Halle-, por medio de la fundación de la Universidad de Halle (1694) ${ }^{7}$. El espíritu liberal de

6 El resultado más destacado de los esfuerzos teóricos de Fichte, W. von Humboldt, Schelling, Schleier y Steffens fue la fundación de la Universidad reformada de Berlín el 10 de octubre de 1810. Los escritos-que continúan siendo hoy de valiosa lectura- de los autores citados sobre la reforma universitaria están reproducidos en Anrich, Ernst (Coord.), Die Idee der deutschen Universität, Darmstadt, 1956.

7 Cfr. KITZINGER, "Die Freiheit der Wissenschaft und Kunst", en: Die Grundrechte und Grundpflichten der Reichsverfassung, Bd. 2, Berlín 1930, pp. 449 y ss. (453). 
la llustración llegó también expresado en las Constituciones de las Universidades de nueva fundación de Göttingen (1734) y Erlangen (1743). El primer Kurator de la Universidad de Göttingen, el Ministro de Estado von Münchhausen, formuló la libertad de cátedra de modo acentuadamente extensivo: "A nuestros profesores les está sin duda permitido explicar por si mismos el Derecho del Estado alemán, exclusivamente según su propia convicción, sin fijarse en si sus tesis doctrinales sirven de soporte a los intereses de aquellas clases (sociales) del Reich a las que nuestro Regente pertenece; o si están de acuerdo o no con los todavía especiales intereses de nuestra Corte»8.

La libertad de cátedra e investigación no fue protegida constitucionalmente en el siglo xvili; permanecía siempre a disposición de la autoridad estatal respectiva. Con acierto define Alexander Kluge ${ }^{9}$ la libertad de cátedra como la expresión característica de un Absolutismo anterior a 1789, seguro de sí mismo, y por lo tanto generoso (liberal).

En la primera mitad del siglo $x \mathrm{x}$, las Decisiones de Karlsbad ${ }^{10}$ representaron un revés para el desarrollo de la libre enseñanza e investigación en las universidades alemanas. La primera Ley de Karlsbad, la «Resolución provisional referente a las Universidades y las medidas que deben tomarse en consideración", de 20 de septiembre de 1819, fue, en contra de su denominación como "provisional", una Ley Federal ilimitada, que no fue derogada hasta la Decisión Federal de 2 de abril de 1848, junto con las correspondientes leyes excepcionales de la Federación.

El §1 de la Ley de Universidades preveía para todas las universidades la figura de un Mandatario gubernamental, que tenía ilimitadas tareas de control. El "comisario estatal" debía desempeñar su función de vigilancia "sin injerencias directas en los métodos científicos o de enseñanza", pero sus competencias para el régimen disciplinario de los estudiantes, así como para el control de la enseñanza -en el sentido de que a ella corresponde el futuro destino de los jóvenes académicos, esto es, la preparación para el servicio del Estado-, podían conducir, a través de una oportuna y rígida interpretación de algunos conceptos jurídicos indeterminados, a la censura de la enseñanza.

p. 475.

8 Citado por RößLER, Emil F., Die Gründung der Universität Göttingen, 1855, 1958, p. 71.

9 KLUGE, Alexander, Die Universitäts-Selbstverwaltung, Frankfurt del Main,

10 Del 6 al 31 de agosto de 1819 se reunieron en Karlsbad los Ministros de diez estados alemanes, que trabajaron sobre los proyectos de cuatro leyes federales. 
Además, la libertad de investigación y enseñanza fue puesta en grave peligro por medio de la amenaza permanente de una espada de Damocles. El § 2 de la Ley universitaria obligaba a los Gobiernos de los Länder a la separación de su puesto de todos los docentes universitarios, cuando estos hubieran mostrado de modo evidente su incapacidad para el ejercicio del importante cargo encomendado, por haber incurrido en una desviación probada de su obligación, o haber sobrepasado la frontera de su profesión; por medio del abuso de su legítima influencia sobre el pensamiento de los jóvenes; por medio de la decisión de doctrinas perjudiciales, contrarias al orden público, o que corrompan el fundamento de las instituciones estatales existentes.

La acumulación de conceptos jurídicos indeterminados en la determinación de los supuestos jurídicamente relevantes, y la falta de cualquier tipo de procedimientos jurídico-estatales para la separación del cargo, abría de par en par la puerta de los abusos. A través de decisiones administrativas inapelables podía ser apartado de su Cátedra cada profesor antipático al Régimen, con lo cual se daba el hecho especialmente grave, de que un profesor apartado de su cargo en su Universidad, no podía volver a trabajar en ningún otro puesto docente de ningún otro Estado alemán"1.

La Ley universitaria deja traslucir inequívocamente un "estilo de redacción austríaco" ${ }^{12}$; la aplicación de la Ley en la práctica varía, no obstante, considerablemente, de un Estado a otro. Especialmente en Prusia ${ }^{13}$

11 Este riguroso principio fue pronto quebrantado por algunos estados individuales, que ante los despidos de profesores practicados en otros Länder, reflexionaron de nuevo sobre su plausibilidad. Por ejemplo, en Jena, en 1819, el filósofo suspendido Jakob Friedich Fries recibió, después de algunos años, una cátedra de matemáticas en su Universidad; Lorenz Oken, especialista en Ciencias Naturales, también despedido en Jena, fue nominado en 1828 en la Universidad de Munich, aunque después se trasladó, en 1832, a Zurich. También los «siete de Gotinga", relegados por motivos políticos, fueron tras algunos años nuevamente nominados para alguna Cátedra fuera de Hannover, o recibieron otro puesto (por ejemplo los hermanos Jakob y Wilhelm Grimm, por iniciativa de Federico Guillermo IV, en la Academia de Berlín).

12 El Memorandum del Bundestag austríaco para la preparación de las decisiones de Karlsbad exigía la eliminación de todos los profesores "dañinos" de las universidades "sin sentencia judicial»; además se constataba que la Universidad como institución de enseñanza y formación está bajo control del Estado. Cfr. ILSE, L. Fr., Geschichte der deutschen Bundesversammlung, Bd. 2, Marburg 1861, pp. 17 y ss.

13 Cfr. Discurso rectoral de August BöcKH, de 3 de agosto de 1847, reproducido en : Idee und Wirklichkeit einer Universität, Berlin 1960, p. 373. 
y Sachsen-Weimar ${ }^{14}$ no tuvo efecto nunca la Resolución de Karlsbad con el posible rigor de su texto.

En Prusia contribuyó a esto decisivamente la posición jurídica de la Ley Universitaria, imponiendo para todas las universidades un Mandatario gubernamental. La Instrucción prusiana para los Mandatarios gubernamentales extraordinarios en la Universidad, de 18 de noviembre de $1819^{15}$, designaba a los Mandatarios gubernamentales como máximos guardianes de la disciplina académica y del espíritu académico de fidelidad al Gobierno y al Estado por parte de los profesores y estudiantes. Además fue transferida al Mandatario gubernamental la función del Kurator, que originalmente debía asumir el Presidente (Ober-Präsident) de cada Provincia para su Provincia universitaria ${ }^{16}$. Los Mandatarios gubernamentales eran al mismo tiempo el más alto cargo administrativo y órgano de control político de su Universidad. Decisivo fue para esto que ellos, como Kurator, estaban directamente subordinados al Ministro pruso de cultura en Berlín, y el Ober-Präsident no tenía ninguna influencia en la Universidad. Para esta organización administrativa era también decisivo qué tipo de indicaciones recibía el Mandatario gubernamental desde Berlín. Para las Universidades prusianas fue una suerte que prevaleciese, en los hombres responsables del Ministerio de Cultura, un espíritu liberal, comprometido con la promoción científica.

El Barón Karl Friedrich von Stein zum Altenstein ${ }^{17}$, durante largos años Ministro de Cultura, afirmaba en relación con la discusión sobre la Decisión de Karlsbad: "La Ciencia tiene en sí misma el remedio para todo lo que puede contribuir a su propia ruina y a la de la Instrucción» ${ }^{18}$.

Para el mantenimiento de un ámbito científico autónomo y libre, en las universidades prusianas se daba la circunstancia de que un Ministro de Cultura liberal elegía al Mandatario gubernamental y le transmitía sus instrucciones confidenciales. Además, fue esencial que parte

14 Cfr. Fritz HaRtung, Das Großherzogtum Sachsen unter der Regierung Carl Augusts 1775-1828, Weimar 1923, pp. 428 y ss.

15 Preußische Gesetzessammlung 1819, p. 233.

16 Conforme al art. 16 del Decreto de 30 de abril de 1815.

17 Con la Orden gubernamental de 3 de noviembre de 1817 fue separada del Ministerio del Interior la sección de Enseñanza y Cultura (con la sección para el sistema Médico), y fue elevada al rango de Ministerio para Enseñanza y Cultura. Altenstein (1770-1840) fue nominado Ministro de Cultura, y ocupó ese difícil cargo hasta su muerte.

18 Reproducido por MüsEBECK, Ernst, Das preußische Kultusministerium vor 100 Jahren, Stuttgart/Erlangen, 1918, p. 277. 
de los estudiantes (Burschenschaften -Corporación de estudiantes-) y un buen número de profesores se mostraban partidarios, con sus palabras y sus hechos, de la tradición liberal de la Universidad alemana. Los valores liberales se mantuvieron vivos -no sólo en Prusia ${ }^{19}$ - hasta la abolición formal de la ley excepcional ${ }^{20}$.

Las experiencias vividas con las inconcretas e indeterminadas medidas de vigilancia durante el tiempo de la Restauración, condujeron en el año revolucionario de 1848 , por primera vez, a una protección legal-constitucional de la libertad científica ${ }^{21}$. Desde la Constitución de Paulskirche (Frankfurt) de 1849 hasta hoy la Constitución federal (alemana) ha determinado que la enseñanza es libre ${ }^{22}$. No obstante, durante el tiempo del Nacionalsocialismo y durante la existencia de la DDR no fue asegurada la libertad de cátedra en su totalidad.

Según el artículo 3 del Einigungsvertrag (Tratado de Reunificación), con el ingreso definitivo de los nuevos Bundesländer y de Berlín Este en la República Federal de Alemania, ha entrado en vigor para ellos la Ley Fundamental de Bonn. Con ello, es válido para toda Alemania el artículo 5, párrafo 3 de la Grundgesetz. La libertad de cátedra está igualmente garantizada en muchas Constituciones de los Länder ${ }^{23}$; la Hochs-

19 También, por ejemplo, en Sachsen-Weimar; cfr. HARTUng, Fritz, Das Großherzogtum Sachsen unter der Regierung Karl Augusts 1775-1828, Weimar, 1923, pp. 428 y ss.

20 «Resolución federal sobre la derogación de la Ley excepcional de 2 de abril de 1848", vigesimoséptima sesión, § 214; a través de los acontecimientos de marzo quedaron fácticamente sin eficacia las leyes excepcionales en la mayoría de los Länder ya antes de la decisión derogatoria de la Asamblea Federal.

21 Cfr. art. 22 de la uLey concerniente a los Derechos fundamentales del pueblo alemán", de 27 de diciembre de 1848 (Bundesgesetzblatt (Boletín Oficial del Estado Federal) de 28 de diciembre de 1848, p. 49).

22 El artículo 152 de la Constitución de Paulskirche dice: "La ciencia y su enseñanza es libre». El artículo 142 de la Constitución de Weimar dice: “El arte, la ciencia y su enseñanza son libres. El Estado garantiza su protección y participa en su cuidado». El artículo 5.3. de la Ley Fundamental de Bonn dice: «El arte, la ciencia y la enseñanza son libres. La libertad de enseñanza no exime de la fidelidad a la Constitución"s.

23 Art. 20.1 de la Constitución de Baden-Württemberg: "La Universidad es libre en la investigación y la enseñanza ". Art. 108 de la Constitución de Baviera: "La ciencia y la enseñanza son libres». Art. 31.1.3 de la Constitución de Brandenburgo: "(1) La ciencia, la investigación y la enseñanza son libres. (3) La libertad de enseñanza no exime de la fidelidad a la Constituciónn. Art. 11 de la Constitución de Bremen: «El arte, la ciencia y su enseñanza son libres. El Estado garantiza su protección y participa en su cuidado". Art. 10 de la Constitución de Hessen: "Nadie puede ser obstaculizado en su creación científica o artística y en la difusión de sus trabajos». 
chulrahmengesetz (HRG, Ley (Marco) federal de Universidades) ${ }^{24}$ y las Hochschulgesetze de los diversos Länder ${ }^{25}$ basadas en ella garantizan asimismo la libertad de cátedra en el plano legislativo ordinario.

\section{LA LIBERTAD DE CÁTEDRA}

\section{Concepto y fundamento de la libertad de cátedra}

El concepto de enseñanza no está definido en el artículo 5, párrafo 3 , frase primera de la Grundgesetz, sino que se da por supuesto ${ }^{26}$. El Bundesverfassungsgericht define la enseñanza como «la transmisión científicamente fundamentada de los conocimientos adquiridos a través de la investigación ${ }^{27}$. De modo similar se define por la doctrina la enseñanza como la transmisión de los conocimientos científicos, por lo tanto como una especial "publicidad de la Ciencia» ${ }^{28}$. Esporádicamente se entiende el concepto de enseñanza de modo restrictivo, en el sentido de considerarla sólo como la divulgación de los propios resultados de la investigación -al menos en las universidades-, y con re-

Art. 7.1 de la Constitución de Mecklenburg-Vorpommern: «El arte, la ciencia y la enseñanza son libres. La libertad de enseñanza no exime de la fidelidad a la Constitución». Art. 9 de la Constitución de Renania-Palatinado: «El arte, la ciencia y la enseñanza son libres. La libertad de enseñanza no exime de la fidelidad a la Constitución". Art. 39.1 y 2 de la misma Constitución: "Las Universidades tienen el derecho a la propia administración. La libertad de investigación y de enseñanza les están a ellas garantizadas». Art. 5.2 de la Constitución del territorio del Sarre: "La cultura, la ciencia y la enseñanza son libres». Art. 33.2, 1 y 2 de la misma Constitución: «Las Universidades tienen el derecho a la propia administración. La libertad de investigación y de enseñanza está garantizada". Art. 21 de la Constitución de Sajonia: «El arte, la ciencia y la enseñanza son libres. La libertad de enseñanza no exime de la fidelidad a la Constitución". Art. 10.3 de la Constitución de Sajonia-Anhalt: "El arte y la ciencia, la investigación y la enseñanza son libres. La libertad de enseñanza no exime de la fidelidad a la Constitución. La libertad de investigación no exime de la atención a la dignidad de las personas y la defensa de los fundamentos de la vida natural». Art. 27 de la Constitución de Turingia: «El arte es libre. La ciencia, la investigación y la enseñanza son libres. La libertad de enseñanza no exime de la fidelidad a la Constitución".

24 Cfr. Art. 3.3 HRG.

25 Cfr., por ejemplo, art. 4.4 de la UG NW.

26 Cfr. KNEMEYER, F. L., Lehrfreiheit. Begriff der Lehre - Träger der Lehrfreiheit, 1969, pp. 9 y ss.

27 BVerfGE 35, 79, 125.

28 Cfr. KNEMEYER (nota 26), pp. 31 y ss. 
ferencia a ello, la enseñanza de profesores de la Universidad tendría que ser distinguida de otras actividades educativas (Anlern-, Wiederholungs- und Ausbildungsveranstaltungen) -actividades introductorias, repetitoria y de formación- desempeñadas por los asistentes de los Catedráticos (wissenschaftliche Mitarbeiter und wissenschaftliche Assistenten $)^{29}$.

En sentido contrario a este restrictivo concepto de enseñanza, hoy es reconocida la libertad de cátedra -en el sentido del artículo 5 , párrafo 3 de la Grundgesetz- a cada docente científico, sin tener en consideración el tipo de vinculación o incorporación a la Universidad ${ }^{30}$.

Elementos sustanciales de la enseñanza son, por tanto, la impartición de clases en el marco de las Universidades, eligiendo el método y contenido, y la expresión pública de las opiniones científicas que está en conexión con dicha tarea; expresión que puede manifestarse también de otras maneras ${ }^{31}$.

La jurisprudencia establece como criterio identificativo de la libertad de cátedra que la tarea docente se lleve a cabo de modo independiente y autónomo ${ }^{32}$. Con acierto ha sido apuntado que las incertidumbres resultantes de lo anterior sólo podrían ser resueltas cuando se reconoce que no sólo existe un magisterio uniforme en las universidades, sino también diferentes funciones docentes de acuerdo con las tareas y el tipo de enseñanza ${ }^{33}$.

La jurisprudencia ha asumido esta idea de que el legislador no está obligado a reunir a todos los docentes en un solo grupo ${ }^{34}$. De acuerdo con la distinta asignación de funciones se ha dicho también que es diferente el margen de actuación de cada docente y su posición en el Claustro de profesores ${ }^{35}$. Por eso, el derecho fundamental de libertad de cátedra no excluye que, por ejemplo, un profesor universitario indique a sus colaboradores las pautas o instrucciones a seguir en la enseñanza que está a su cargo ${ }^{36}$.

29 Cfr. Evers, H.-U., en: "Beiträge zum Hochschulrecht", Beiheft 4, WissR 1970, pp. 47 y ss.

30 Cfr. KNEMEYER (nota 26), pp. 7 y ss.; BVerfGE 35, 79, 125.

31 Cfr. VG Arnsberg, KMK-HSchR 1987, 126, 127 y 128.

32 Cfr. VGH BW, ESVGH 25, 144 y ss.; OGV Berlin, DVBI 1972, 738.

Rdnr. 57.

HaIlbronner, Kay, en: Hailbronner (Coord.), Kommentar zum HRG, § 3 ,

HessVGH, KMK-HSchR 1987, 129, 130.

Así, Hailbronner (nota 33), § 3, Rdnr. 57.

Así, acertadamente, HallBronNer, ibid. 
El BVerfG tiene en cuenta también, en el marco del concepto de profesor universitario por él desarrollado, la distinta asignación de funciones en la enseñanza, para así distinguir los profesores cualificados para la tarea universitaria de los profesores cualificados de otra manera.

EI BVerfG entiende como profesor universitario (en sentido material) sólo aquél investigador o docente a los que, a través de la oposición a una cátedra universitaria (Habilitation) u otra prueba de cualificación, se les encomienda una materia científica de enseñanza e investigación, con autonomía propia ${ }^{37}$; por lo cual, los profesores que realizan exclusivamente su labor en los estudios de las Escuelas Técnicas Superiores (Fachhochschulstudiengängen), por causa de su diferente cualificación y función, así como de las consecuencias que de ello se derivan, no son considerados como profesores universitarios, teniendo en cuenta el concepto de Universidad en sentido material (wissenschaftliche Hochschulen) ${ }^{38}$.

Por la doctrina ${ }^{39}$ se ha intentado negar la diferencia mostrada por el BVerfG entre la enseñanza de los profesores universitarios, por un lado, y la de los profesores de Escuelas Técnicas Superiores (Fachhochschulen) por otro. La uprueba" para sostener la igual calidad de la enseñanza universitaria y la de Escuelas Técnicas Superiores viene tomada fundamentalmente a partir de las advertencias sobre las prescripciones generales de la Hochschulrahmengesetz, en la que, al contrario de lo sostenido por el BVerfG, no son tenidas en cuenta en la mayor parte de los casos las características cualitativamente distintivas (diferencias entre estudios universitarios y los de Escuelas Técnicas Superiores; diferente cualificación de cada una de las categorías de profesores).

Por consiguiente, si bien la cualificación de la enseñanza de las universidades y las Escuelas Técnicas Superiores no es idéntica, está sin embargo fuera de duda que también los profesores de Escuelas Técnicas Superiores son titulares del derecho a la libertad de cátedra en el marco de las funciones a ellos encomendadas.

Del artículo 5, párrafo 3, frase 1 (art. 5.3.1) de la Grundgesetz (GG) no se permite deducir que corresponda a una Facultad un derecho

37 Cfr. BVerfGE 43, 368.

38 Cfr. BVerfGE 61, 210, 242 y ss.; cfr. en este sentido también Waldeyer, "Das Recht der Fachhochschulen", en Hailbronner (coord.), Kommentar zum HRG, Rdnrn. 30 y ss.

39 Cfr. WALdeyer (nota 38 ), Rdnrn. 30 y ss. 
autónomo constitucionalmente protegido para determinar de modo exclusivo la dimensión y el contenido de la oferta educativa y llevarla a cabo de modo autónomo.

El art. 5.3.1 GG no contiene tampoco ninguna garantía de estabilidad en la asignación de determinadas materias de enseñanza a los profesores. Más bien se deja al criterio de organización política del legislador la tarea de asignar las concretas materias de enseñanza a las distintas facultades o segregar una parte de un ciclo de estudios de una Facultad para atribuirla a otra ${ }^{40}$. Esta precisión no afecta a la libre actuación en la ciencia, investigación y enseñanza de aquellos habilitados por los derechos fundamentales ${ }^{41}$.

\section{2. Ámbito de la libertad de cátedra}

La doctrina y la jurisprudencia definen la libertad de cátedra como la libre elección de objeto, forma, método y contenido, así como la libertad (protección) ante cada injerencia externa en dichos ámbitos ${ }^{42}$.

El núcleo constitucional sustancial de la libertad de cátedra se manifiesta en el derecho a verse libres de recibir ${ }^{43}$ instrucciones oficiales que se refieran al modo de llevar a cabo y al contenido de la enseñanza.

También la elección de método pertenece al núcleo sustancial de la libertad de cátedra, que la jurisprudencia deduce del ámbito del criterio científico-pedagógico de los profesores, el cual sólo puede ser examinado judicialmente ${ }^{44}$.

En el artículo 3.3.1 de la Hochschulrahmengesetz son enumerados a modo de ejemplo los más importantes elementos de la libertad de cátedra ${ }^{45}$.

40 Se refiere a que un profesor no puede exigir siempre impartir una determinada (y la misma) materia. Cfr. BVerfG, KMK-HSchR 1984, 854, 859; BVerfGE 15, 256, 264.

${ }_{41}$ Cfr. BVerfG, KMK-HSchR 1984, 854, 859.

42 Cfr. OVG Berlín, WissR 1969, 178; así también Thieme, Deutsches Hochschulrecht, 1956, p. 243; más claramente, del mismo autor, Hochschulrecht, 2. edición, 1986, Rdnr. 66 y ss.

43 La traducción literal sería "derecho a no recibir".

44 Cfr. VG Karlsruhe, KMK-SchR 1982, 348; VGH BW, NJW 1984, 1832; VG Hannover, KMK-SchR 1985, 678, 679.

${ }_{45}$ Con esta enumeración debe ser señalada la protección absoluta y sin reservas del ámbito de la libertad que a todos los profesores corresponde y que no 
El derecho al ejercicio de actividades docentes está orientado a su desarrollo efectivo sin obstáculos y a la libertad de contenidos y métodos didácticos en el modo de llevarla a cabo. De la libertad de cátedra no se deduce ninguna protección ante la competencia; una actividad educativa en competencia puede ser ofertada también sin el consentimiento de los otros docentes ${ }^{46}$.

La autonomía de los docentes en el ejercicio de actividades académicas incluye también todas las decisiones no reguladas legalmente, ya sea a través de normas sobre exámenes y estudios, o por medio de la exigencia de unos requisitos necesarios para asegurar el ejercicio cotidiano de la enseñanza ${ }^{47}$.

A dicho ámbito pertenece, entre otros, el habitual anuncio de actividad educativa pública universitaria, incluso el derecho a escoger o determinar el nombre bajo el que se desarrolla la actividad docente (nombre de la asignatura) y, posiblemente, un texto de explicación de dicho título ${ }^{48}$.

El derecho a llevar a cabo actividades docentes incluye la puesta a disposición de las aulas apropiadas para su ejecución, así como los medios de apoyo necesarios dentro de las posibilidades existentes. La organización autónoma de la enseñanza supone que se ha de tener en cuenta sobre todo, dentro del ámbito de las posibilidades de organización, la propuesta de los profesores para el lugar y hora de su realización ${ }^{49}$.

En caso de dificultades o irregularidades, el derecho al ejercicio de la enseñanza garantiza la protección de la actividad educativa ${ }^{50}$. El ejercicio de dicha actividad incluye también el certificado de participación en la actividad docente (Bescheinigung der Teilnahme an Lehrveranstaltungen), y la evaluación de los rendimientos obtenidos dentro

debe ser sometida a la toma de decisiones (así la exposición de motivos gubernamental, BT-Drucks 7/1328, 33). Nota: Aunque en el texto original se cita el artículo, como es habitual en Alemania, de otro modo ( $\$ 3$ Abs. 3 Satz 1), lo hemos adaptado al modo habitual de citar en nuestro país.

46 Cfr. BVerwGE 20, 235 y ss. Nota: El texto se refiere a la posibilidad de que dos profesores impartan materias que se solapen, o que coincidan en sus programas docentes.

${ }_{47}$ Cfr. LÜTHJE, en Denninger (coord.), Kommentar zum HRG, 1984, § 3, Rdnr. 30.

48 LÜthJE, en el lugar citado.

49 Cfr. LüTHJe (nota 47), 53 Rdnr. 31.

5o Cfr. para ello BVerfGE 35, 128; 55, 68; PIERoth, Störung, Streik und Aussperrung in der Hochschule, 1976, pp. 230 y ss. (con más detalle). 
de ella. Estos elementos constituyen el aspecto más personal de la libertad de cátedra como derecho del docente ${ }^{51}$.

El derecho al ejercicio de la actividad docente no incluye la autorización para dejar de celebrar o interrumpir las actividades docentes anunciadas. Esto sólo se permite en los casos excepcionales de imposibilidad o de que no pueda ser exigible el desarrollo de la actividad ${ }^{52}$.

Como los estudiantes tienen el derecho de participar en las actividades docentes libremente elegidas, no se deduce del derecho a ejercer actividades docentes el derecho a decidir sobre los participantes ${ }^{53}$. En el caso de seminarios de investigación, los profesores tienen el derecho de elegir a los participantes, teniendo en cuenta las condiciones necesarias para una colaboración fructífera ${ }^{54}$.

El diseño de contenidos y métodos abarca en especial la forma de la descripción de la materia de enseñanza, su preparación y presentación ideológica y metódica. En atención al derecho fundamental individual de libertad de cátedra, las normas reguladoras de los estudios y las decisiones de los órganos de las universidades deben permitir a los profesores un ámbito libre de actuación para el diseño de método y contenidos. De los diferentes tipos de actividad docente (lecciones introductorias, lecciones de profundización, seminarios, clases prácticas, etc.) se derivan algunas limitaciones para los profesores, con respecto a la fijación de método y contenidos.

A través de las normas reguladoras de los estudios son determinadas además el tipo y la dimensión de cada materia de enseñanza. La libertad para fijar el contenido se puede por lo tanto desarroliar en una lección sobre el ámbito de los derechos fundamentales en la Constitución, de tal manera que el profesor puede decidir en qué orden puede presentar él la materia, sobre qué derechos fundamentales debate más en profundidad y qué medios de apoyo emplea para la transmisión de conocimientos ${ }^{55}$. Por el contrario, no está protegido por la libertad de cátedra que se hable durante todo el semestre en una asignatura semejante sólo sobre la dignidad humana, sin que se debata de ninguna manera sobre el resto de la materia.

51 Cfr. LÜTHJE (nota 47), \$ 3, Rdnr. 33.

52 Cfr. ConRAD, "Rechtsprechungsbericht", WissR 1979, 68 y ss.; HAILBRONNER, Die Freiheit der Forschung und Lehre als Funktionsgrundrecht, 1979, pp. 233 y ss.

53 Cfr. VG Friburgo, NJW 1970, 1762 y 1763.

54 Cfr. LÜTHJE (nota 47), § 3 Rdnr.34.

55 Cfr. Hallbronner (nota 33), 83 Rdnr. 61. 
El derecho a expresar opiniones doctrinales científicas constituye el núcleo del ámbito de libertad garantizado sin reservas. Comprende no sólo una presentación sin restricciones de las propias convicciones científicas de los docentes o de sus propios resultados de la investigación, sino también el derecho a presentar y criticar los resultados de la investigación y las opiniones científicas de otros investigadores. La libertad para sostener opiniones científicas o un determinado concepto de ciencia es válida para todos los docentes científicos, también si ellos llevan a cabo las actividades docentes de acuerdo con el método elegido, siguiendo las directrices e instrucciones ${ }^{56}$.

\section{Planificación, Coordinación y Organización de la actividad docente}

La enseñanza está sujeta en el ámbito de la Universidad a diversas vinculaciones, que encuentran su raíz en diferentes y complejas regulaciones. Por la jurisprudencia ha sido constatado que los órganos académicos no están autorizados a influir de un modo determinado sobre los métodos y contenidos de la actividad docente ${ }^{57}$. Por otro lado, cada profesor tiene que someterse a diferentes restricciones, relacionadas con la modalidad de su enseñanza.

La organización de la actividad de enseñanza trae consigo para cada profesor algunos límites, que se derivan de las limitaciones de aulas y medios materiales. La libertad de cátedra garantizada como derecho fundamental en el art. 5.3.1 GG protege también la libertad de organización de la enseñanza, que es en primer lugar un derecho de cada uno de los profesores y subsidiariamente un derecho de las unidades de organización competentes de cada Universidad (Departamentos/Facultades).

Con acierto ha subrayado por ello el Tribunal Administrativo de Friburgo ${ }^{58}$ que le está prohibido al Tribunal, por causa de la libertad de organización de la enseñanza garantizada por el art. 5.3 GG, imponer una totalmente determinada organización de la enseñanza a la Facultad contra la que se recurre.

En concordancia con el art. 43 de la HRG, las leyes universitarias de los Länder ${ }^{59}$ establecen que los profesores desempeñan sus tareas de modo autónomo.

Cfr. LütHJe (nota 47), § 3 Rdnr. 38.

Cfr. VG Hannover, KMK-HschR 1985, 678, 679 (con más detalle).

Cfr. VG Friburgo, KMK-HSchR 1979, 419.

Cfr., p. ej., art. 48.1 de la UG NW. 
La realización práctica de la libertad de cátedra exige medidas de organización que, junto al anuncio universitario habitual de las clases, otorga principalmente un derecho exigible ante la Universidad a una adecuada aula en un tiempo oportuno, y a una cesión de los medios necesarios ${ }^{60}$.

Es indiscutible que las tareas de enseñanza de las Universidades están necesitadas de una regulación al respecto; por una parte tiene que ser coordinada de modo organizado la tarea de enseñanza de un gran número de profesores, por otra parte debe ser asegurada la misión formativa de la Universidad ${ }^{61}$.

Del mandato del art. 12 HRG y de la regulación legal respectiva de cada Land ${ }^{62}$-de las que se deriva el aseguramiento de la oferta educativa de acuerdo con las normas reguladoras de los estudios de cada Universidad (Studienordnung), para garantizar una adecuada ordenación de los Estudios (Studium) así como el cumplimiento del tiempo de estudio regulado ${ }^{63}$ - se sigue respectivamente para los profesores universitarios la obligación de armonizar la oferta educativa desde el punto de vista temporal, material y local ${ }^{64}$.

Junto a ello, corresponde a la propia iniciativa y coordinación autónoma de los profesores -con preferencia ante las posibilidades legalmente previstas ${ }^{65}$ - una tarea educativa delegada por la Facultad ${ }^{66}$. Que ésta se reconoce a la propia iniciativa y coordinación autónoma de los profesores, antes que a una forzosamente determinada transmisión de la tarea docente, se deriva directamente de la libertad de cátedra garantizada por el art. 5.3 GG y del consecuente mandato de respetar el principio de proporcionalidad en el caso de las escasas posibles injerencias, y en este sentido se regula legalmente ${ }^{67}$ de modo esporádico y expreso como sigue:

"Si no puede ser alcanzado bajo el amparo de las obligaciones del profesor ningún acuerdo sobre la distribución y aceptación de la actividad docente, la Facultad les transmite a ellos, en el marco de

Cfr. OVG NW, NWVBI 1992, 98, 99.

Cfr. Avenarius, WissR 1980, pp. 51 y ss.

Cfr. p. ej. art. 86 de la UG NW.

Cfr. art. 10, párrafos 2-4 de la HRG, art. 84 de la UG NW.

Cfr. VG Arnsberg, KMK-HSchR 1987, 1,2.

Cfr. p. ej., art. 86.3 de la UG NW.

Cfr. HessVGH, WissR 1987, 72, 73; 74, 78.

Cfr. art. 86.3 de la UG NW. 
las normas aplicables a su relación de servicio, las tareas necesarias para garantizar la oferta educativa requerida».

De lo anteriormente dicho resulta que la organización de la enseñanza es, en primer lugar, un derecho de cada docente, así como un derecho de los docentes en cuanto miembros de una Facultad o de un concreto ámbito de enseñanza, con el fin de alcanzar una coordinación autónoma.

Bajo la condición de que el plan de estudios sea cubierto por la actividad docente y no se produzcan coincidencias entre asignaturas obligatorias, queda a criterio de los profesores la distribución temporal de la actividad docente, sin que haya ninguna base jurídica para una intervención de los órganos colegiales o incluso del Estado.

Para garantizar una atención plenamente responsable de la oferta de enseñanza de una Facultad, los órganos académicos pueden examinar si existen lagunas en el plan de clases que necesiten ser colmadas (por ejemplo, una denominada clase obligatoria no ofertada $)^{68}$. Eventualmente pueden también en casos semejantes ser confiadas determinadas tareas a los profesores universitarios, a cuya función docente corresponde la suplencia en las materias afectadas $^{69}$.

El deber de enseñar de los profesores comprende también la obligación de llevar a cabo las actividades docentes previstas por la normativa sobre los estudios de cada Universidad para las asignaturas que imparten; la enseñanza no consiste en un objetivo científico autónomo, sino que sirve particularmente a la tarea formativa de las Universidades $^{70}$. Las Universidades sólo pueden desempeñar su tarea de formación legalmente prevista cuando los profesores desarrollan en sus asignaturas la enseñanza requerida para la realización de unos Estudios conforme a lo previsto en el ordenamiento ${ }^{71}$.

Para la asignación de tareas de enseñanza adicionales debe ser ciertamente tenido en cuenta que la Universidad interviene con ello en el círculo del derecho individual del profesor funcionario, que se ve afectado así por dicha instrucción de servicio en su libertad de cátedra, 229,230 .

Cfr. BVerwGE 20, 239.

Cfr. art. 12.2 de la HRG.

Cfr. Hailbronner (nota 33), \$ 3 Rdnr. 64.

71 Cfr. OVG Lüneburg, VerwRspr. Bd. 24, 774; HessVGH, KMK-HSchR 1987, 
y eventualmente puede proceder contra dicha medida judicialmente, puesto que se trata de un acto administrativo ${ }^{72}$.

En ningún caso se permite que sea enlazada con el compromiso de explicar unos determinados temas, en el marco de actividad docente, una concepción preconfigurada (Präjudizierung) sobre las opiniones científicas de los profesores o una limitación del diseño de los contenidos o métodos ${ }^{73}$.

La libertad de cátedra viene también limitada a través del derecho de los estudiantes a un objeto y método científico, una formación orientada al ejercicio de una profesión, así como a través de la libertad de aprendizaje o de estudio.

Los profesores universitarios y otros docentes habrían incumplido sus obligaciones cuando ellos, apelando al art. 5.3.1 GG, presentasen y enseñasen de modo subjetivo cualquier materia, de cualquier forma, apartándose de los planes de estudios y normativas de exámenes, así como evadiendo el directorio de clases (Vorlesungsverzeichnis) de la Universidad, y separándose de la acostumbrada y permanente praxis académica en sus lecciones, clases prácticas y seminarios ${ }^{74}$.

Frente a las infracciones graves de cada docente contra esas obligaciones, especialmente frente a las desviaciones importantes de las normas de cumplimiento obligatorio sobre el plan de estudios y normativa de exámenes, puede ser incoado un proceso por el órgano de la Universidad competente contra el profesor o el colaborador cientifico afectado (sobre los que recae la responsabilidad de la actividad); en cada caso concreto deben ser tenidos en cuenta, para resolver la cuestión de los medios empleados, las fronteras derivadas del art. 5.3.1 GG y el principio de proporcionalidad ${ }^{75}$.

También la competencia para examinar de los docentes está comprendida por el derecho fundamental del art. 5.3.1 GG ${ }^{76}$. No obstante, existen siempre determinadas características de cualificación para desarrollarla.

A un profesor que trabaja en un ciclo de estudios integrado en una Escuela Universitaria (Gesamthochschule), que no posee el perfil

72 Cfr. HessVGH, KMK-HSchR 1987, 233, 235.

73 Cfr. BVerwGE 20, 235 y ss.

74 Cfr. Kimminich, WissR Beiheft 4, 1970, 119, 127.

75 Cfr. VG Berlin, WissR 1979, 76.

76 Discutible; cfr. BayVGH, DÖV 1985, 496 y 496; OVG Berlin, DVBI 1985, 1088-1089. 
de exigencia de una cualificación científica a través de una Habilitación o una prueba semejante, le debe ser cerrada la participación como examinador en un Doctorado (Promotion) ${ }^{77}$.

En lo que se refiere a los exámenes, controles de rendimiento, obtención de certificados, etc., teniendo en cuenta la igualdad de condiciones de los exámenes, está permitida una normativa más amplia sobre los requisitos del procedimiento ${ }^{78}$.

De la separación funcional entre la actividad de examinar y de enseñar se deriva que un profesor universitario, que tiene según la ley universitaria del Land la tarea profesional de participar en los exámenes estatales a tenor de la normativa de exámenes, estaría incumpliendo el deber al que como examinador está obligado, si él alega que sólo está dispuesto a examinar candidatos que al menos puedan presentar un certificado de rendimiento (Leistungsnachweis) obtenido con dicho profesor ${ }^{79}$.

La enseñanza científica está condicionada de modo permanente por las prescripciones relativas a la capacidad y la determinación del tiempo de enseñanza. A través de las prescripciones de capacidad puede ser regulado el tamaño de los grupos de alumnos para determinados tipos de actividad docente.

Con acierto ha sido sin embargo apuntado que la frontera llegaría allí donde se produjesen situaciones insoportables para la ejecución materialmente adecuada de una actividad docente, que hiciera a los profesores imposible desarrollar su tarea formativa prevista a través de la regulación de los estudios, conforme a su concepción de los contenidos y los métodos ${ }^{80}$.

De la fijación del tiempo de enseñanza depende en qué medida puede el profesor dedicarse a su segunda tarea principal, la investigación. La jurisprudencia se ha tenido que ocupar con frecuencia de la cuestión de la determinación del tiempo de enseñanza ${ }^{81}$. Así se ha establecido, por ejemplo, que no es justificable una reducción del tiempo

77 Cfr. BVerwG, KMK-HSchR 1988, 1097, 1100.

78 Cfr. Hailbronner (nota 33), § 3 Rdnr. 69.

79 Cfr. OVG Berlin, KMK-HSchR 1986, 222, 223.

80 Cfr. Hallbronner (nota 33), Rdnr. 70; cfr. globalmente sobre el derecho de capacidad Bahro/Berlin/Hübenthal, Das Hochschulzulassungsrecht, Kommentar, tercera edición, 1994.

${ }_{81}$ Cfr. OVG Lüneburg, KMK-HSchR 1987, 909; BayVGH, KMK-HSchR 1988, 155; OVG NW, WissR 1986, 257; HessVGH, KMK-HSchR 1987, 138; HessVGH, KMKHSchR 1987, 171. 
de enseñanza sólo porque un profesor sea miembro de una comisión examinadora o de otros gremios universitarios ${ }^{82}$.

Se ha hecho notar acertadamente que la general y excesiva acentuación de las tareas docentes lleva consigo una serie de cargas sobre la actividad investigadora, como consecuencia de unos programas cada vez más sobrecargados, lo cual conduce a una restricción de la libertad de investigación garantizada constitucionalmente ${ }^{83}$.

Resulta poco convincente determinar esquemáticamente la distribución porcentual de la dedicación a la investigación y a la docencia para conseguir un hipotético rendimiento completo del trabajo universitario ${ }^{84}$. De ello se deduce que la investigación y la docencia no permiten ser rigurosamente separadas la una de la otra; la investigación recibe estímulos y propuestas desde la actividad docente, de la investigación se derivan impulsos directos para la docencia ${ }^{85}$.

Sin embargo, existe un límite de gravamen hacia los profesores universitarios en lo que se refiere a su actividad docente y administrativa, cuya superación conduce a una lesión de la libertad de investigación desde el punto de vista del Derecho, y que permite sostener una división equilibrada entre investigación y docencia ${ }^{86}$.

\section{La reserva de la cláusula de fidelidad}

El art. 5.3.2 GG proclama: "La libertad de cátedra no exime de la lealtad a la Constitución". Según una parte de la doctrina, el art. 5.3.2 GG no contiene, estableciendo el compromiso de la enseñanza ante la Constitución, ninguna nueva barrera; esa prescripción debe más bien aclarar mejor solamente el contenido del art. 5.3.1, según el cual la libertad de ciencia no comprende la actividad politica ${ }^{87}$.

El sentido de esta norma constitucional ha sido determinado por Carlo Schmid en el Consejo parlamentario de la manera siguiente:

82 Cfr. BayVGH, KMK-HSchR 1988, 155, 159.

83 Cfr. Hallbronner (nota 33), § 3 Rdnr. 71.

84 Cfr. el "Informe de la Comisión de Universidades de la Conferencia de Ministros de Cultura sobre Determinación de las obligaciones docentes", NVwZ 1985, 552, 555; cfr. además BVerfGE 61, 210; BVerwG, KMK-HSchR 1989, 172, 177; OVG NW, WissR 1986, 257, 260.

85 Cfr. Hallbronner (nota 33), § 3 Rdnr. 71.

86 Cfr. Hallbronner, en el lugar citado.

87 Así, Merten, BayVBI. 1978, pp. 554, 558. 
luego el art. 5.3.2 «no debe bajo ningún concepto excluir una crítica consciente y responsable a la misma Constitución y también a los principios sobre los que se basa; él sitúa exclusivamente una crítica semejante bajo la obligación de la responsabilidad y el respeto que se debe a una ley aprobada por un pueblo para vivir de acuerdo con ella. La norma debe impedir que, bajo el pretexto de una crítica científica, desde la Cátedra sea emprendida una política insidiosa, que no critica la democracia y sus instituciones, sino que las hace despreciables: sea este un aviso a todos aquellos que pretendieran intentar sabotear científicamente la democracian ${ }^{88}$.

De esta cita se han extraído en los escritos diferentes deducciones. Mientras según algunos autores el art. 5.3.2 GG limita sólo las actividades políticas de la enseñanza, otros quieren situar esa norma sólo en el ámbito de la actividad en el exterior de la Universidad ${ }^{89}$. La doctrina dominante ${ }^{90}$ refiere, por el contrario, la obligación de lealtad al contenido de la enseñanza misma. El art. 5.3.2 GG está en estrecha relación con la "fortalecida democracia" (wehrhafte Demokratie) protegida por los artículos 9.2, 18 y 21.2 .

La cuestión de si la cláusula de lealtad sólo aclara las fronteras que de antemano son extraídas de la enseñanza ${ }^{91}$, o de si se trata de una obligación fundamental ${ }^{92}$, debería tener un significado teórico más que práctico.

Si bien el art. 5.3.2 GG protege efectivamente la democracia y sus instituciones, esto no significa que esa norma prohíba la crítica neutral a disposiciones singulares o a los principios de la GG, sino "sólo... el comportamiento desleal contra el ordenamiento básico libre y democráticon" ${ }^{93}$.

Si existen razones fundadas para suponer que un solicitante de una nominación en la Universidad abusa de su libertad de cátedra para luchar contra el ordenamiento fundamental libre y democrático, ésta puede justificar el rechazo de la Universidad a la propuesta de nominación ${ }^{94}$.

88 JöR N.F. 1 (1951), 92.

89 Cfr. al respecto, ScHolz, Rupert, en: Maunz/Dürig, GG Kommentar (Comentarios a la Grundgesetz), art. 5.3 GG, Rdnr. 198.

90 Cfr. Denninger, en: Alternativ-Kommentar, 2. edición, 1989, art. 5.3.1 de la GG, Rdnr. 44.

91 Cfr. Merten (nota 87), p. 558.

92 Así, Stoвer, Grundpflichten und Grundgesetz, 1979, pp. 32 y ss.

93 Así, Scholz (nota 89), Art. 5 Abs. 3 Rdnr. 199.

94 BVerwGE 52, 313, 318. 
También el encargo docente se sitúa por principio bajo la obligación de lealtad, con independencia de cómo esté previsto en el derecho de los funcionarios el tipo de relación legal aplicable al encargo docente, con lo cual la obligación de lealtad se trata de una función específica ${ }^{95}$. Esta obligación de lealtad a la GG no debe confundirse con una lealtad al Gobierno ${ }^{96}$.

\section{CUESTIONES JURÍDICAS SOBRE LA EVALUACIÓN DE LA ENSEÑANZA}

De modo acertado, Hufen ${ }^{97}$ describe, en 1995, el fundamento actual para las diversas actividades de evaluación de la enseñanza, de la manera siguiente: después de los notorios fracasos de los intentos -a través de presiones directas o indirectas sobre los estudiantes- para lograr un acortamiento del tiempo de duración de los estudios, no pocos políticos universitarios están fijando desde hace años su atención sobre los profesores.

Sin tener en cuenta las causas sociales y económicas de la duración del tiempo de estudios, $y$ evadiendo los problemas que con el aumento del número de estudiantes, el estancamiento de los medios de las Universidades, pero también con la cuestión acerca de la capacidad de estudio de una gran parte de los estudiantes afectados; ha sido desde algún tiempo percibida como causa de los reales o hipotéticos síntomas de crisis de la enseñanza universitaria, la competencia didáctica de los docentes universitarios o la ausencia de disponibilidad por parte de ellos para que la enseñanza se desarrolle adecuadamente.

Así se ha convertido la "mejora de la calidad de la enseñanza" en una frase hecha de la discusión sobre política educativa, que se puede encontrar no sólo entre los objetivos establecidos por las políticas educativas de todos los partidos, sino que también ha encontrado ya influencia en distintos programas de acción, decisiones de las Conferencias de Ministros de Cultura y de Primeros Ministros de los Länder,

95 Cfr. BVerwGE 81, 212, también en JZ 1989, 437, 439, con notas marginales do Kimminich.

96 Cfr. VG Berlin, NJW 1989, 1688, 1690.

97 Cfr. HufEN, Rechtsfragen der Lehrevaluation an wissenschaftlichen Hochschulen, 1995, p. 3. 
notificaciones de los Ministerios, así como en leyes y proyectos de ley ${ }^{98}$.

Estos esfuerzos tienen en común alcanzar una mejora de la enseñanza académica a través de una mayor competitividad y motivación, parcialmente (pero también) a través de una presión directa y de sanciones.

El fundamento sería, al menos parcialmente, una comparación superficial con las experiencias americanas, especialmente en relación con la evaluación de la enseñanza y el "ranking" 99 . Se da por supuesta, además, la condición de que hay considerables defectos en el ámbito de la enseñanza, que pueden ser solucionados a través de la evaluación y sus consecuencias.

La opinión jurídica sobre la evaluación de la enseñanza presupone que las diversas formas y finalidades de la evaluación son claramente diferenciadas. Hay que distinguir según los sujetos: estudiantes, institutos externos, (alguna) Facultad o Universidad, el Estado; así como según los instrumentos: encuesta, procedimiento, la evaluación misma, publicación y sanción ${ }^{100}$.

En la doctrina se ha subrayado que es común a las diferentes formas de evaluación que intentan medir el "éxito" de la enseñanza con baremos que proceden, por regla general, del exterior, y por tanto son heterónomos. Este sería el modo de referirse a la enseñanza científica ${ }^{101}$.

Si la evaluación proviene de estudiantes o grupos de estudiantes -no de órganos de las corporaciones de estudiantes (Studentenschaften) constituidas- no se tratará por lo general de ninguna injerencia en la libertad de enseñanza ${ }^{102}$. También la publicación está protegida por el derecho fundamental de libertad de expresión y de prensa, siempre que no se trate de grabaciones realizadas secretamente, de la publicación de falsificaciones de hechos y citas, así como de la difusión de insultos personales ${ }^{103}$.

Si un Ministerio de la Ciencia asume, por el contrario, los resultados de la evaluación de los estudiantes, que pasa a ser así la

98 Cfr. la considerable muestra de Acciones, Programas, etc., en Hufen (nota 97), nota 1.

99 Cfr. al respecto RaU, WissR 1988, pp. 210 y ss.; sin embargo, más escéptico, Rupp, H. H., WissR 1988, pp. 138 y ss.

100 Asi, acertadamente, HuFEN (nota 97), p. 44.

101 Hufen (nota 97), en el lugar citado.

102 Cfr. Hufen (nota 97), pp. 15-19.

103 Cfr. Hufen (nota 97), pp. 20-22. 
evaluación estatal, las autoridades estatales se sirven de resultados ajenos para fines propios. Teniendo en cuenta los conocidos defectos de los métodos de la evaluación de los estudiantes, resulta obvia la infracción del principio del Estado de Derecho ${ }^{104}$. El Estado no debe hacer propios ningunos datos de los que no conoce bien su procedencia.

El principio del Estado de Derecho no pretende sólo determinar un ordenamiento en libertad a través de la concesión de derechos subjetivos y del establecimiento de la división de poderes, sino que pretende asignar también competencias y funciones estatales institucionalmente fortalecidas y racionalmente constituidas.

La estructura estatal debe ser organizada de modo ordenado, claro y de acuerdo con el principio de responsabilidad; y no de cualquier manera, sino de tal modo que "las tareas estatales y las decisiones también son desarrolladas y tomadas por órganos que, según sus estructuras internas, ocupación, forma de trabajo, procesos de decisión

104 Los defectos de las «Evaluaciones» comparativas según el modelo de Examen a los profesores son, entre otros, los siguientes: las Evaluaciones conducen a la valoración a través de los destinatarios de una encuesta que, con frecuencia, no disponen por sí mismos de una cualificación especial en la materia para poder juzgar sobre el contenido de una actividad docente; apenas pueden evitar preguntas con respuestas ya predeterminadas; no miden la calidad de la enseñan$\mathrm{za}$, sino el grado de aprecio a la materia y al profesor; desde el punto de vista de la libertad de estudio no aparece ninguna obligación relativa a la asistencia continuada a clases; el resultado de la Evaluación se basa frecuentemente sólo en visitas esporádicas a la actividad docente; el éxito o fracaso de los trabajos escritos se incluye también en la Evaluación; no hay ningún baremo unitario para medir la buena enseñanza; se tiene en cuenta antes la cualidad según el criterio de los estudiantes, con preferencia a las formas de la enseñanza, es decir, sobre todo la relevancia de los exámenes, los cuadernos repetitorios de enseñanza con muchos escritos, los cuales difícilmente pueden ser calificados como enseñanza científica; premian una enseñanza complaciente, impregnada de efectos divertidos, liberada de transmisiones de pensamiento; comparan funciones, ámbitos y actividades docentes no susceptibles de comparación; dan por supuesto que todos los estudiantes son igualmente competentes, así que la mayoría de las afirmaciones se consideran como acertadas; se incluyen factores de la calidad de la enseñanza sobre los que los docentes no tienen responsabilidad (trabajos en periodos de vacaciones, estructura social de las corporaciones de estudiantes, formación educativa previa, aulas inapropiadas, en muchos casos excesiva ocupación de las aulas, etc.); también la simpatía o aversión contra determinadas manifestaciones de la enseñanza influyen en la valoración; finalmente, algunos estudiantes utilizan el anonimato para permitirse una broma con el profesor, por medio de contestaciones inadecuadas o desacertadas. Cfr. al respecto, también, ScHOLz, Oskar Berndt, Evaluation von Lehrveranstaltungen durch Studenten, Forschung \& Lehre 1995, 497 y ss. 
observados, etc., están legitimados y estructurados para realizar esta tarea, tomando decisiones eficaces» ${ }^{105}$.

Es obvio que un Ministerio infringe el principio del Estado de Derecho cuando los resultados de una evaluación de los estudiantes son convertidos en el fundamento de decisiones que afectan a la relación jurídica de servicio de los funcionarios, o que tienen consecuencias financieras.

Los preceptos legales y los decretos ministeriales prevén también la evaluación a través de la misma Facultad o Universidad, es decir, por medio de una decisión jurídico-administrativa del propio titular. Como medida relevante en el campo de los derechos fundamentales, la evaluación necesita un fundamento jurídico, respecto a cuya procedencia constitucional no se ha dicho todavía nada.

Puesto que el contenido y el método de la actividad docente no pueden estar sujetos a una evaluación estatal ${ }^{106}$, la evaluación sólo puede aplicarse sobre el cumplimiento de las obligaciones docentes externas. Dejando aparte la situación excepcional de los procedimientos de nombramiento de un profesor (Berufungsverfahren), según el derecho (constitucional) sobre universidades vigente, ningún profesor está sometido a la valoración por parte de otros colegas o de expertos de fuera de la Universidad, ya que a ninguno de ellos corresponde una posición superior con competencia para juzgar. Los profesores universitarios -esto parece haber caído en el olvido- gozan de una posición libre de la posibilidad de recibir órdenes y dictámenes.

Una evaluación a través de la autoridad estatal competente en ese ámbito significa, independientemente de las sanciones de ella derivadas, una infracción contra la libertad de recibir órdenes y dictámenes que corresponde a los profesores universitarios, y por ello también es constitucionalmente inadmisible. Sólo es lícito el control del "cumplimiento externo" de las obligaciones docentes ${ }^{107}$.

Como ya la evaluación estatal es contraria al derecho, tampoco pueden ser decididas en consecuencia sanciones como la privación de medios, la denegación de semestres de investigación, el rechazo de las negociaciones sobre la permanencia, etc.

105 Ossenbühl, DÖV 1980, 545, 549.

106 Cfr. VG Karlsruhe, KMK-HSchR 1982, 348; VGH BW, NJW 1984, 1832; VG Hannover, KMK-HSchR 1985, 678, 679.

107 El cumplimiento del tiempo de enseñanza determinado es una obligación de servicio natural; el control del "cumplimiento externo" de las obligaciones docentes no es conceptualmente ninguna evaluación. 
En conjunto, debe ser constatado que la evaluación de la enseñanza es un camino equivocado. La enseñanza universitaria deb́ mantenerse plural y exigente, no debe ser transformada en una enseñanza única a través de criterios pedagógicos fijos, sobre todo cuando la $\mathrm{Pe}$ dagogía y la Didáctica están sometidas a cambios permanentes. Además debe ser claramente afirmado que en la Universidad existe una clara y exigente actividad docente, que muchos estudiantes no pueden superar; pero que aquellos dotados de talento e inteligentes reciben a través de la Universidad un bagaje de conocimientos sustancial para sus caminos venideros.

Las altas cuotas de fracaso en los ámbitos de las ciencias matemáticas y naturales, o en Ingeniería, no hablan automáticamente de una ausencia de "Calidad de enseñanza", sino quizá solamente de que no se puede conseguir que todo estudiante asimile la materia de enseñanza -incluso con la mejor enseñanza-. 